\title{
INJURIES TO THE KNEE JOINT
}

SIDNEY SACKS, F.R.C.S., Orthopaedic Surgeon, Johannesburg.

Before describing the various injuries that may occur to the knee joint it is essential to mention the important role played by the Quadriceps extensor apparatus.

This apparatus is the most important single factor in the maintenance of the erect posture in man, and in maintaining an efficient knee joint.

A weak or wasted Quadriceps predisposes to injury of the knee joint, and an injured knee joint gives rise to rapid wasting of the Quadriceps. This wasting creates a greater predisposition to further injury to the joint, and a vicious circle is thus established, viz. : Injury $\rightarrow$ Effusion $\rightarrow$ Rest $\rightarrow$ Quadriceps wasting

The principle therefore to be rigorously observed in the treatment of nearly all injuries to the knee joint, is to do everything possible to maintain the tone in the Quadriceps extensor apparatus throughout the process of healing and repair of the-injury.

Injuries to the knee joint can be classified as follows :-

(i) Injuries of the synovial membrane.

(ii) Injuries of the ligaments.

(iii) Injuries of Menisci.

(iv) Injuries of the Extensor apparatus.

(v) Injuries of the bones.

(vi) OTHER conditions in the knee, related to trauma. 
(I) INJURIES OF THE SYNOVIAL MEMBRANE

(a) Traumatic Synovitis. This is due to injury to the soft tissues of the joint. A fringe of synovial membrane is contused or torn. The joint space becomes filled with effusion.

Clinical Features.-There is swelling of the joint and a patellar tap is present.

'Treatment.-If the diagnosis of primary traumatic synovitis has been made, and secondary synovitis due to either internal derangements of the knee, bony injury, or tearing of the ligaments has been excluded, an attempt should be made to limit the effusion by applying a tight bandage to the knee. If there is excessive swelling, the joint should be aspirated and a back splint is advisable. Quadriceps drill is started straight away. The splint may be discarded in a few days, and weight bearing allowed. Recovery should be complete in a few weeks.

(b) Recurrent Synovitis. This is usually due to inefficient quadriceps protection. The cure lies in remedying this defect. During this period weight bearing should be limited.

The Differential Diagnosis lies between:

(i) Internal derangement of the knee

(ii) Tuberculosis of the knee.

(iii) Syphilis.

These must be excluded in every case.

(c) Traumatic Haemarthrosis. This is characterised by almost immediate swelling of the joint. The joint is tenser and more painful, and firmer than in the case of a synovitis. There is pyrexia due to absorption.

Causes.-(I) Tearing of a vessel in the synovial membrane.

(2) Fracture of the bones entering into the formation of the joint.

(3) Tearing of the coronary ligament.

(4) Injury to an osteoarthritic joint

Treatment.-The blood should be aspirated, and a tight elastic bandage applied. Immobilisation on a back splint is necessary for two weeks. Quadriceps drill should be practised during, this period so as to prevent wasting of this muscle.

\section{(II) INJURIES OF THE LIGAMENTS}

(a) Sprain of Internal Collateral Ligament. Only a few fibres are torn and the stability of the joint is unimpaired.

Treatment.-

(I) Firm elastic bandage to knee.

(2) Raising the inner side of the heel and sole $1 / 4$ inch to prevent valgus strain.

(3) Quadriceps drill.

(b) Rupture of the Internal Collateral Ligament. This is due to over-abduction of the extended knee. The ligament may be torn :

(I) From its origin on the condyle of the femur.

(2) From its insertion to the tibia.

(3) Opposite the joint space in the region of the semilunar cartilage.

Clinical Features.-Rupture of the ligaments is characterised by swelling, bruising, and local tenderness over the point of injury. There is undue mobility of the tibia which can be abducted as much as $20^{\circ}$ to $30^{\circ}$.
Treatment.-When the ligament is torn either at its origin or insertion, the knee should be immobilised for at least eight weeks in a plaster cast extending from the ankle to the upper thigh. The knee should be almost fully extended and the tendency to abduction fully corrected. Quadriceps clrill should be assiduously practised during the whole of this period. If the ligament is torn in the region of the cartilage, the chances of healing with a good result are much less, since there is a tendency for the ruptured ends to curl into the joint. In these cases operative suture is advised. Complications.-(I) Pellegrini Stieda's Disease.This is ossification in a subperiosteal haematoma occurring at the origin of the ligament.

(2) Instability of the Joint with Consequent Recurrent Synovitis.-Every effort should be made to restore the tone of the quadriceps. If this is achieved the symptoms will in most cases disappear. If this fails, reconstruction of the internal lateral ligament by means of the semitendinosus tendon should be considered.

(c) Rupture of the External Lateral Ligament. This ligament is usually torn at its insertion into the styloid process of the fibula.

Clinical Features.-All the signs of traumatic internal lateral ligament. The opposite deformity. namely increased adduction of the leg occurs.

Treatment.-As for internal lateral ligament tears, but the tendency to varus deformity is corrected. Untreated cases or cases in which treatment has failed do not give rise to as much trouble as on the inner side, and for this reason operative reconstruction of the ligament from the ilio-tibial tract is seldom necessary.

Complication.-Contusion of the peroneal nerve.

(d) Rupture of the Cruciate Ligaments. The anterior cruciate ligament runs laterally and posteriorly to the medial aspect of the lateral condyle of the femur. It is usually torn as the result of hyperextension, but may be torn in over-abduction.

Clinical Features.-All the signs of traumatic synovitis or haemarthrosis are present, together with excessive forward mobility of the tibia on the femur.

The posterior cruciate ligament runs from the back of the tibial spine forwards and medially to the lateral aspect of the medial condyle of the femur. It may be torn by violence which pushes the tibia back on the femur.

Clinical Features.-The signs of synovitis together with undue backward mobility of the tibia on the femur.

Treatment.-Complete imobilisation of the $\operatorname{limb}$ from the toes to the groin is required.

For tears of the anterior cruciate ligament, the knee is flexed to about $30^{\circ}$ and the tibia pushed backwards, whereas in the case of the posterior cruciate ligament the tibia is pulled forward. This is maintained for three months.

Quadriceps drill must be assiduously practised during all this time. For neglected or failed cases, operative reconstruction of the ligaments has been performed, but the results have not been impressive.

\section{(III) INJURIES OF THE MENISCI.}

(a) The Medial Meniscus. This is torn as the result of a rotatory strain imposed on the semi-flexed and abducted knee during weight bearing. The femur rotates medially on the fixed tibia or the 
tibia externally on the femur. The following lesions may occur :

(J) A longtitudinal split of the cartilage causing a bucket handle tear.

(2) The coniplete cartilage may be torn from the capsule.

(3) A tag from the anterior horn.

(4) A tag from the posterior horn.

(5) A tag from the middle of the cartilage.

(6) Displacement of the posterior horn.

The first two lesions cause locking of the joint. The rentainder cause sensations of instability but no actual locking.

Clinical Features.-The main points in the diagnosis are :

(J) A history of an injury such as has been described

(2) Pain and often the sensation of tearing on the inner aspect of the knee.

(3) Locking of the knee with inability to extend it completely.

(4) The appearance of a traumatic synovitis a few hours after the injury. This gradually subsides over a period of several weeks.

(5) Spontaneous unlocking may occur but manipulation may be necessary.

Examination during the acute stage shows the signs of traumatic synovitis with the added signs of ( $\mathrm{J}$ ) Locking of the knee, (2) Tenderness over the joint line anterior to the collateral ligament. The $X$-ray shows no bony injury.

At a later stage the only physical sign may be tenderness over the medial meniscus. Further attacks are liable to follow, but in each subsequent attack the traumatic synovitis is less marked. Finally a stage may be reached where the cartilage is displaced, and replaced by the patient without any other symptoms.

Localised tears of the cartilage produce a feeling of insecurity in the knee, but locking does not occur. A sensation of something slipping in the joint may be described, but localisation is vague. A most important clinical sign is McMurray's sign. To elicit this the knee is fully flexed. The foot is now extcrnally rotated and the leg abducted. In this position the knee is slowly extended. As the angle alters, the loose tag of semilunar cartilage is caught between the condyles of the tibia and fomur. and as the femoral condyle slides over the tag, there is a click and the patient conılains of pain. The degree of flexion indicates how far back the tear is. A similar effect may be produced by a lax cartilage, but the click is not audible and there is no pain.

Differential Diagnosis.-(i) Enlarged Infra-patellar Pad of Fat.-This pad of fat is extra-articular and is liable to contusion in extension of the joint. Normally the subcrural muscle pulls on the synovial membrance and drags the alar pad of fat out of the joint during extension. Inefficiency of this nutuscle will result in nipping of the pad with resulting contusion, haemorrhage, granulation tissue formation and organisation.

Clinical Features.-Nipping of the pad is followed by acute pain behind the patella or its ligament. There may be an accompanying synovitis. Examination shows tenderness on either side of the ligamentum patellae, and inability to completely extend the knee. There may be palpable thickening of the pad.
Treatment.-In early cases quadriceps drill is necessary to restore the extensor mechanism of the knee to its full efficiency. The heel is raised $1 / 4$ inch so as to prevent full extension of the knee. In late cases the enlarged pad of fat should be removed. Quadriceps drill must still be undertaken.

(ii) Fractured Tibial Spine.

(iii) Loose Bodies.-These may be present without giving rise to symptoms. If, however, they slip into the articulating regions, they cause locking of the joint, pain, and traumatic synovitis. Varieties.-

(a) Fibrinous : Soft, structureless bodies resulting from the organisation of exudate associated with synovitis or haemarthrosis.

(b) Fibrous: Resulting from hypertrophy and isolation of synovial fringes.

(c) Cartilaginous: Arising from the menisci or articular cartilage or chondrification in the synovial membrane.

(d) Osteocartilaginous: The result of fractures either of bone or marginal osteophytes, or osteochondritis dessicans.

Diagnosis.-Sometimes the loose body can be felt. Radio-opaque bodies can be visualised by X-ray. Treatment.-Loose bodies which are giving rise to symptoms should be removed. The underlying condition must also be treated.

(b) The Lateral Meniscus. This is torn less frequently than the internal cartilage. The injuring force is an adduction, internal rotation strain of the tibia on the femur. The pain and tenderness is on the outside of the joint. Actual locking may occur, but more frequently audible clicks on extending the knee are the main symptoms.

\section{Treatment of Injuries to Menisci.}

It is customary to treat the first injury by reducing the dislocated cartilage, and then immobilising the knee for a month on a back splint. If the cartilage is torn from its attachment to the capsule it will become reattached after reposition, since there is a good blood supply in this region. In the case of longtitudinal tears of the cartilage itself, the injury is through an avascular part and healing does not take place. Recurrence of symptoms is therefore most likely. For recurrent symptoms the only treatment is complete removal of the cartilage. Failure to do this will ultimately lead to osteoarthritis of the joint.

\section{(IV) INJURIES OF THE EXTENSOR APPARATUS}

The extensor mechanism of the knee joint comprises the Quadriceps, the patella, the ligamentum patellae and the tubercle of the tibia.

The quadriceps tendon may be torn at its insertion into the patella; the patella may be fractured transversely or in a comminuted fashion; the ligamentum patellae may tear at its upper or lower attachment; and the tubercle of the tibia may be partially separated or completely avulsed from the tibia in adolescents.

Treatment.-If the quadriceps tendon or the ligamentum patellae are only partically torn, immobilisation on a back splint for 4 weeks is necessary and then quadriceps exercises are commenced.

If they are completely torn, they should be sutured.

Fractures of the patella are treated either by plaster immobilisation, or repair by operation, or in 
some cases the patella is completely excised. After a period of 3 weeks, quadriceps exercises are commenced and gradual active flexion of lithe knee encouraged.

Injuries to the tibial tubercle are treated by reduction and immobilisation of the knee in plaster in a fully extended position for 6 weeks. Quadriceps tone is maintained throughout this period.

\section{(V) INJURIES OF THE BONES}

(a) Supracondylor Fractures of the Femur. These may or may not involve the articular surface. Accurate reduction is necessary and the position maintained by traction, plaster or internal screws until the fracture is united.

Maintenance of quadriceps tone is essential throughout the treatment.

(b) Fractures of the Tibial Spine. This injury is frequently associated with a tear of the cruciate ligament or the medial meniscus. The joint fills up with blood and the knee cannot be fully extended. If there is displacement of the fragment, the knee is fully extended under an anaesthetic and plaster applied. Quadriceps exercises are practised and the plaster removed after 8 weeks. If reduction cannot be effected, operative replacement is sometimes undertaken.

(c) Fractures of the Tibial Condyles. These are generally intra-articular and may or may not be associated with tears of the collateral ligaments and menisci. These fractures are reduced by closed or open methods and the knee immobilised. Attention to quadriceps exercises is essential while the leg is in plaster.

\section{(VI) OTHER CONDITIONS IN THE KNEE RELATED TO INJURY}

(a) Osteochondritis Dessicans. This is a condition characterised by the flaking off of one or several portions of the articular surface of the joint usually from the medial femoral condyle. It is presumed to be due to poor blood supply to a small area of the joint surface following on injury. The piece of cartilage may eventually separate entirely and become a loose body in the joint. Treatment. - In early cases, immobilisation of the knee in plaster for 8 weeks is tried. If the flaked off area has separated and is acting as a loose body it should be removed by operation.

(b) Prepatellar Bursitis. An inflammation of the bursa in front or below the patella. This inflammation is extra-articular and is treated by rest and chemotherapy. If it becomes chronic, the bursa is excised.

(c) Baker's Cysts. These are bursae occurring in the vicinity of the knee joint and are often in communication with the joint. Their real origin is obscure, but they are frequently associated with osteo-arthritic knee joints.

If they give rise to discomfort they should be removed by operation.

(d) Loose Bodies, have been described above.

(e) Cyst of the meniscus. This usually occurs on the outer edge of the lateral meniscus and may give rise to pain.

The cyst together with the lateral meniscus is excised by operation.

(f) Injuries to the Infra-patella Pad, lave been described above.

\section{CONCLUSION}

It will be noted that in almost every injury to the knee joint, emphasis is laid on the treatment of the quadriceps mechanism.

The physiotherapists have therefore an extremely important part to play in all these injuries - to guide and instruct the patient in maintaining the tone of the quadriceps so that when the ligament, bone, tendon or joint has recovered from its acute phase of trauma the patient will be able to maintain his gait, posture and knee joint movement, with confidence and without fear of recurrent effusion or weakness in the joint. 